\title{
APPLICATIONS OF GAME- THEORY IN ACTIVE STRATEGIC PORTFOLIO MANAGEMENT- THE CASE OF HEDGE - FUNDS ADAPTATION FOR THE REAL CONSTRAINTS IN ROMANIAN CAPITAL MARKET
}

\author{
Eugen-Silviu VRĂJITORU*, Mircea BOSCOIANU*, Elena-Corina BOSCOIANU** \\ *6"Transilvania" University of Brașov, Romania \\ **INCAS Bucharest, Romania \\ eugen.vrajitoru@unitbv.ro; boscoianu.mircea@yahoo.com; boscoianu.corina@incas.ro
}

\begin{abstract}
The application is focused on strategies for portfolio management in the case of hedgefunds for emerging markets taking into account the severe constraints for a real-world implementation. In the case of Romanian capital market, the design of a hedge-fund architecture should respond to the typical constraints for using alternative strategies. Beyond the liquidity problems there exits only a limited set of alternative instruments and strategies with impact on diversification, on the functionality and efficiency. The objective is to develop a better understanding of alternative actions and innovations for real adaptation of the architecture of a hedge-fund at these emerging market conditions, especially the lack of short and hedging instruments and the liquidity problems. Based on this new innovative framework that could capture the value of multiple rotating satellite sub-portfolio paradigm, as an active strategy, it is possible to build a different paradigm for active portfolio management in a dynamic manner. Based on an adequate dynamic of rotation of these sub-portfolios it results an optimal risk-return-liquidity profile for the whole hedge-fund portfolio, adaptable for different contexts.
\end{abstract}

Keywords: hedge-fund, game-theory, active strategic portfolio management (ASPM), sub-portfolio dynamic rotation management (SPDRM)

\section{Introduction}

Although hedge-funds could offer better opportunities in emerging markets due to the extended set of alternative strategies engaged, there are some critics related to the real-world implementation. Indeed, the hedge-fund architectures should be adapted to the typical constraints in emerging markets (constraints due to the small set of instruments for diversification, the functionality of short and hedging instruments, the liquidity gap, the investment culture) [1], [2]. The aim of this contribution is to understand the actions and innovations for an adaptation of the architecture of a hedge-fund in order to offer an efficient implementation. Although hedge-funds can use an extended set of strategies, in the case of emerging markets there exists some constraints attributed to the limited diversification and the liquidity. In this proposal we develop a new framework for understanding the actions in multiple rotating sub-portfolio strategies based on game-theory applied in investment strategies. Beyond the conventional subportfolios with stock and bonds we configure two new types of sub-portfolios: venture capital/ private equity funds (VCFs/ PEFs) a long-term component capable to offer higher returns but constrained by liquidity aspects, and exchange traded 
funds (ETFs), an optimal risk-return investment with a very good liquidity [3], [4]. Based on the dynamics of rotation of these sub-portfolios it results an optimal risk- return profile for the whole hedgefund portfolio. The result is a better valuation of the competitive strategies for rotating sub-portfolios according to the targeted risk- return objective in different contexts. In addition, we will analyze the first mover advantage in picking low liquidity-high return stocks and also cooperative strategies in different market opportunities. This contribution is usefully for understanding the implementation actions for a new hedge- fund in different market contexts, but also for academics and researchers in the field of the dynamics of capital markets, especially in the case of emerging markets.

\section{Aspects of active portfolio management in emerging markets}

The first approach in portfolio management was developed by Markowitz (1952) with focus on trade-off between return and risk (expressed by variance of return). After this start, Sharpe (1963) continued research on portfolio management and through the CAPM (Capital Asset Price Management) model, it was possible to understand the risk-return link in case of a diversified portfolio with an expected residual return considered zero [5]. Subsequently, the research was focused on determining portfolios related to an index equipped with very low fees and near zero operational costs. The basic hypothesis refers to the socalled quasi-universal allocation of all portfolios in fact an index allocation but with the objective of obtaining, a superior performance called active return and characterized by additional active risk. This is an intuitive way to define the concept of active management and in this case, the following elements are defined:

- active return - as the difference from the performance of the reference market index;
- alpha - the expected (active) return;

- omega- active risk.

William Sharpe in his contribution "The arithmetic of active management", (1991) argued based on some classic assumption that active management could offer better performances in portfolio management [6]. The concept of information ratio presented by Grinold (1989) was considered the most important element in the idea of active portfolio management and is related also on the relationship between active management and zero-sum game paradigm, the links between information ratios and other specific elements (efficiency, value added, risk allocations), and most important, the relationship between alpha management and control and other critical elements (volatility, expectations) [7]. Grinold set of assumptions, that active management could be associated to the aggregate of portfolios. Sharpe described the impact of high information ratio products on value added, but also the relation between risk preferences and portfolio allocations. The basic assumption of Sharpe in the concept of active management is related to the fact that broad market index (fund) positions sum the global market (this is true with two mentions related to the exception is the case of small- cap indices and the investors with odd allocations/ positions).

More important is the extension of information ratio for active strategies IRAS, expressed by different indicators like information coefficient (IC) as a measure of investment decision, breadth/ skill (BR-SK) as a measure of diversification or transfer coefficient (TC) as a measure of market efficiency.

3. The concept of active portfolio management for the sub-portfolio dynamic rotation management (SPDRM) The concept of active portfolio management could be applied also to subportfolio dynamic rotation management (SPDRM) taking into account the paradox 
of active management in relation with zerosum game, information ratios impact on efficiency, value added and risk allocations, and SP- alpha control.

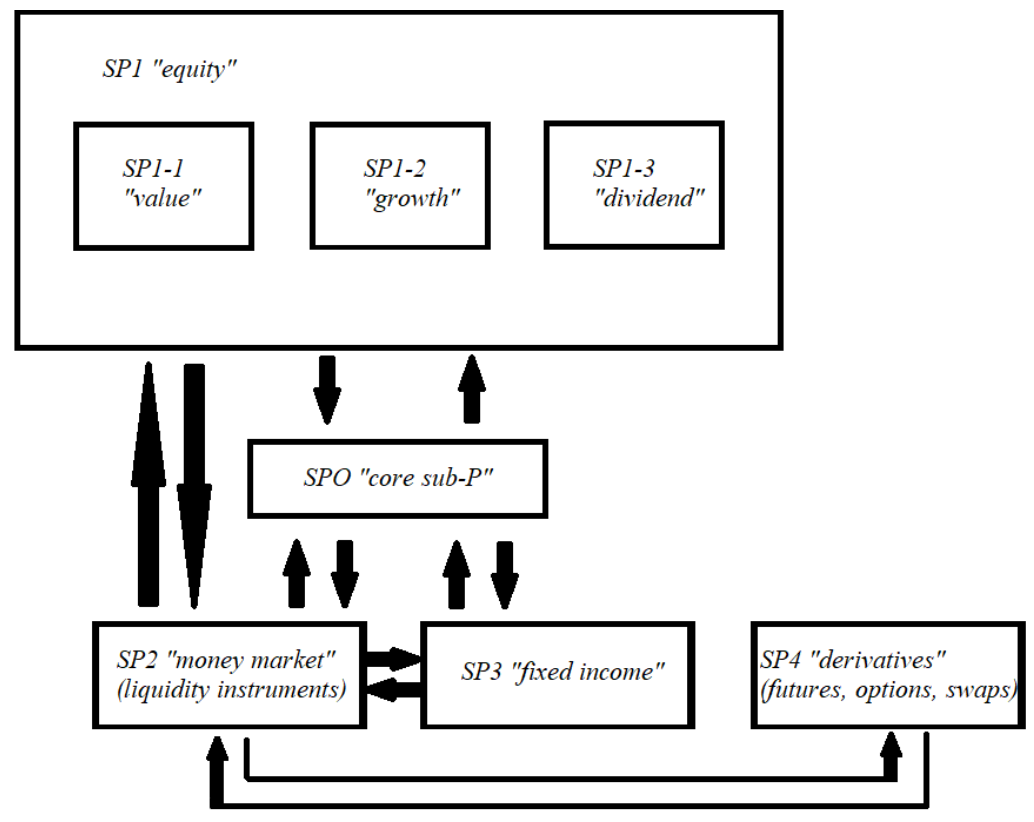

Figure 1: A hedge-fund architecture based on SPn rotation paradigm

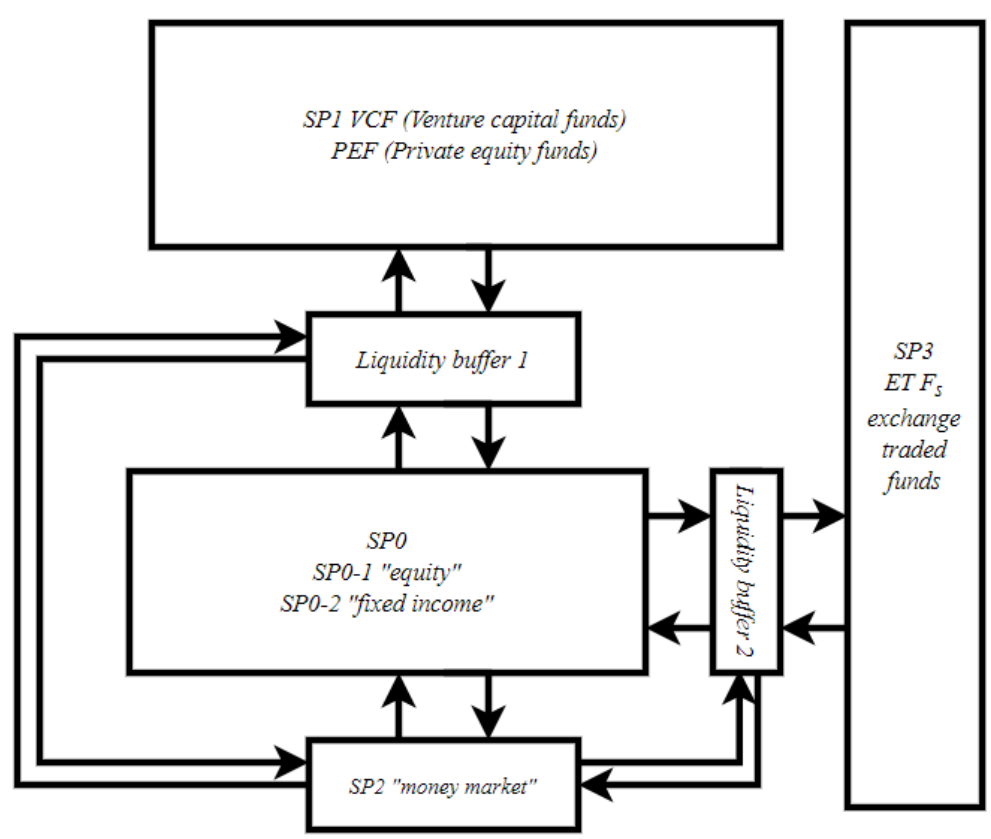

Figure 2: The adopted architecture for emerging markets equipped with liquidity buffers

a) Redefine the concept of active management in the context of rotational sub-portfolio approach

The law of active management underlines the relationship between these indicators and is essential in the assumption that active management beats the market. In the Sharpe paradigm of active management, the average portfolio manager beat the market indices. This aspect is also related to the 
alpha control, a very complex concept (for example in Fama-French paradigm are considered both size, value and momentum factors) that could be adapted and simplified by considering a competition and a ranking of the best performing portfolio managers [8]. Although being above the average player can be a solid argument supported by the index approach, we must keep in mind that there are many cases with alpha negatives. In addition, expected returns should respond to the arguments related to risks and additional costs [9].

b) More implications for information ratios in the case of sub-portfolio management (IR-SPM)

In the paradigm of active portfolio management, the aim is to design and control portfolios with alpha-control (the marginal return should be understood related to the residual risk). The added value is expressed by the utility function of portfolio and takes into account the vector of sub-portfolios (SP1,SP2,..,SPn), the covariance matrix for sub-portfolios CMSP, a risk- aversion factor and a global measure for risk. In markets there are different riskreturn approaches so different risk positions and an expression of risk aversion can be constructed by utility (as a function of risk). This results in the level of residual risk accepted by investors (optimal residual risk as a critical point depends also on information ratio IR). It results also the link between active management capability and information ratios

c) Matching between risk and information ratio in sub-portfolios

In the particular case of mean-variance maximization the aim is to maximize the utility function (based on alpha and risk). It results that the optimal allocation of subportfolios is according the information ratios IRs divided by risk (a simple allocation based only on proportions or rates of IRs). This is very important for the dynamics of sub-portfolio rotation with critical implications for diversification process and global portfolio performance.

d) Particularities for alpha control of sub-portfolios

Based on the relevant information and an adequate control of expectations and volatility it is possible to build intuitive relations with alphas of sub-portfolios (the expected return based on relevant information)

\section{e) The analysis of the impact of costs of transaction}

Based on the transfer coefficient TC it is possible to understand the impact of transaction costs in the case of active portfolio management. It is expected that TC to be inversely proportional with the residual risk. In sub-portfolio rotation management, the costs of adjusting different types of allocations could be significant and could also impact the global performance of the portfolio.

\section{Conclusions}

Most works in literature refer to index performance analyzes. In the case of a portfolio with rotating sub-portfolios it is possible to optimize the risk-returnliquidity of a portfolio without complex strategies based on derivatives [10], [11], [12]. This application is very useful in the case of Romanian capital market because short sales and leverage are not possible. The aim of this contribution was to analyze the real possibilities to build and actively manage a portfolio with rotating satellite sub-portfolios in a dynamic manner. In the case of active portfolio management, we design an ex-ante relationship (based on a targeted return is possible to anticipate the risk or omega). In this approach the performance of a portfolio based on subportfolio rotation anticipate the critical movements of different markets or components. 


\section{References List}

[1] Prelipcean, G., Boscoianu, M., „Risk analysis of a hedge fund oriented on sustainable and responsible investments for emerging markets", „Amphitheatre Economic”, Volume: 22, Issue: 55, Pages: 653-667, DOI: 10.24818/EA/2020/55/653, Published: AUG 2020.

[2] Prelipcean, G., Boscoianu, M., 2020, „An Innovative Flexible Investment Vehicle Oriented to Sustainability - The Adaptation of Hedge Funds in the Case of Emerging Markets”, p. 493-503, „Proceedings of the ENTRENOVA - ENTerprise REsearch InNOVAtion Conference", Virtual Conference, 10-12 September 2020.

[3] Prelipcean, G., Boscoianu, M., 2019, „Aspect Regarding the Design of Active Strategies for Venture Capital Financing - the Flexible Adjustment for Romania as a Frontier Capital Market”, „Proceedings of the ENTRENOVA - ENTerprise REsearch InNOVAtion Conference", Rovinj, Croatia, 12-14 September 2019, pages 187-196, IRENET - Society for Advancing Innovation and Research in Economy, Zagreb.

[4] Prelipcean, G., Boscoianu, M., Lupan, M., Nastase, C.E., „Innovative financing solutions based on Venture Capital and Private Equity to support the development of entrepreneurship in Romania”, „Transformations in Business \& Economics13”, 2014

[5] Sharpe, F.W.,„A Simplified Model for Portfolio Analysis”, „Management Science”, 1963.

[6] Sharpe, F.W., „The Arithmetic of Active Management”,,Financial Analysts Journal”, 1991.

[7] Grinold, R.C., „The Fundamental Law of Active Management”, „Journal of Portfolio Management 15", 1989.

[8] Fama, E.F., French, K.R., „Luck Versus Skill in the Cross-Section of Mutual Fund Returns”, „Journal of Finance 65”, 2010;

[9] Clarke, R., de Silva, H., Thorley, S., „Portfolio Constraints and the Fundamental Law of Active Management”,,Financial Analysts Journal”, 2002.

[10] Boscoianu, M., Prelipcean, G., Lupan, M., „Innovation enterprise as a vehicle for sustainable development - A general framework for the design of typical strategies based on enterprise systems engineering, dynamic capabilities, and option pricing”, „Journal of Cleaner Production” vol 172, 2018.

[11] Boscoianu, M., Prelipcean, G., „The creation of flexible special purpose vehicles (PPPEquity Fund) architectures for stimulating the innovative SMES”, „Business Excellence Challenges during the Economic Crisis", vol 1, pp. 82-85, 2012.

[12] Boscoianu, M., Ceocea, C., Vladareanu, V., Vladareanu, L., „Special purpose vehicles for sustainable finance of innovation in Romania - the case of intelligent robotic systems”, „Periodicals of Engineering and natural sciences” vol 8 no 3/ 20204 A, ISSN 2303-4521, http://dx.doi.org/10.21533/pen.v8i3.1518. 